\title{
The Sampson Laplacian on Negatively Pinched Riemannian Manifolds
}

\author{
Vladimir Rovenski*, Sergey Stepanov and Irina Tsyganok
}

(Dedicated to the memory of Prof. Dr. Aurel BEJANCU (1946 - 2020))

\begin{abstract}
We prove vanishing theorems for the kernel of the Sampson Laplacian, acting on symmetric tensors on a Riemannian manifold and estimate its first eigenvalue on negatively pinched Riemannian manifolds. Some applications of these results to conformal Killing tensors are presented.
\end{abstract}

Keywords: Riemannian manifold, Sampson Laplacian, spectral and vanishing theorems, conformal Killing tensor.

AMS Subject Classification (2020): Primary: 53C20 ; Secondary: 53C25;53C40.

\section{Introduction}

Let $(M, g)$ be a Riemannian manifold. We regard it as a connected $\mathrm{C}^{\infty}$-manifold $M$ of dimension $n \geq 2$ endowed with a metric tensor $g$ and the Levi-Civita connection $\nabla$. Let $T M:=T^{(0,1)} M$ (resp., $T^{*} M:=T^{(1,0)} M$ ) be its tangent (resp. cotangent) bundle where $T^{(p, q)} M=\left(\otimes^{p} T^{*} M\right) \otimes\left(\otimes^{q} T M\right)$. Let $S^{p} M$ (resp. $\left.\Lambda^{r} M\right)$ be a subbundle of $T^{(p, 0)} M$, consisting of covariant symmetric $p$-tensors (resp. differential $r$-forms) on $M$. Denote the vector spaces of their $\mathrm{C}^{\infty}$-sections by $\mathrm{C}^{\infty}\left(T^{(p, q)} M\right), \mathrm{C}^{\infty}\left(S^{p} M\right)$ and $\mathrm{C}^{\infty}\left(\Lambda^{r} M\right)$, respectively.

The Lichnerowicz-type Laplacian has the form (see $[17,24])$

$$
\Delta_{L} T=\bar{\Delta} T+t \Re_{p}(T)
$$

for any $t \in \mathbb{R}$ and $T \in \mathrm{C}^{\infty}\left(\otimes^{p} T^{*} M\right)$. In (1.1), $\bar{\Delta}$ is the Bochner (rough) Laplacian and $\Re_{p}$ is the Weitzenböck curvature operator, which in a known way depends linearly on the Riemann curvature tensor and the Ricci tensor of $(M, g)$. In addition, the Weitzenböck curvature operator of $\Delta_{L}$ satisfies the following identities (see [20, p. 315])

$$
g\left(\Re_{p}(T), T^{\prime}\right)=g\left(T, \Re_{p}\left(T^{\prime}\right)\right)
$$

and

$$
\operatorname{trace}_{g} \Re_{p}(T)=\Re_{p-2}\left(\operatorname{trace}_{g} T\right)
$$

for any $T, T^{\prime} \in \mathrm{C}^{\infty}\left(\otimes^{p} T^{*} M\right)$. In particular, for $t=1$, (1.1) yields the formula $\Delta_{L}=\bar{\Delta}+\Re_{p}$ of the ordinary Lichnerowicz Laplacian (see [20]; [1, pp. 53-54]). We recall that the formula

$$
\Delta_{H} \omega=\bar{\Delta} \omega+\Re_{p}(\omega)
$$

for an arbitrary $p$-form $\omega \in \mathrm{C}^{\infty}\left(\Lambda^{p} M\right)$ determines the well known Hodge Laplacian (see [1, p. 35]; [25, pp. 335; 347]). At the same time, the Sampson Laplacian $\Delta_{S}$ acting on $\mathrm{C}^{\infty}$-sections of the vector bundle $S^{p} M$ has the following Weitzenböck decomposition (see [24, 28, 33]):

$$
\Delta_{S} \varphi=\bar{\Delta} \varphi-\Re_{p}(\varphi)
$$

for any $\varphi \in \mathrm{C}^{\infty}\left(S^{p} M\right)$. Therefore, the differential operator $\Delta_{S}$ is also an example of the Lichnerowicz-type Laplacian for the special case when $t=-1$ and $T \in \mathrm{C}^{\infty}\left(S^{p} M\right)$. 
Formulas of the type (1.1) are particularly important in the study of interactions between the geometry and topology of Riemannian manifolds. In fact, there exists a method, due to Bochner, of proving vanishing theorems for the null space of a Laplace operator admitting the Weitzenböck decomposition and furthermore of estimating its lowest eigenvalue (see [1, pp. 52-53]; [25, pp. 333-364]). This method mainly applies to compact manifolds. As an application of the Bochner technique, we recall the following theorem in [41]: If $(M, g)$ is a closed (i.e., compact and without boundary) Riemannian manifold with positive (resp. negative) curvature operator of the second kind, $\stackrel{\circ}{R}: S_{0}^{2} M \rightarrow S_{0}^{2} M$, then it does not admit the Hodge Laplacian $\Delta_{H}$ with the non-degenerate null space, hence the Betti numbers $b_{1}(M)=\ldots=b_{n-1}(M)=0$ (resp. the Tachibana numbers $t_{1}(M)=\ldots=t_{n-1}(M)=0$, that we conclude from [35]).

Remark 1.1. The Riemann curvature tensor $R m$ induces an algebraic curvature operator $\stackrel{\circ}{R}: S_{0}^{2} M \rightarrow S_{0}^{2} M$ (see, for example, [19]). The symmetries of $R m$ imply that $\stackrel{\circ}{R}$ is a selfadjoint operator, with respect to the point-wise inner product on $S_{0}^{2} M$. That is why, the eigenvalues of $\stackrel{\circ}{R}$ are all real numbers at each point $x \in M$. Thus, we say $\stackrel{\circ}{R}$ is positive semidefinite (resp. positive-definite), or simply $\stackrel{\circ}{R} \geq 0$ (resp. $\stackrel{\circ}{R}>0$ ), if all the eigenvalues of $\stackrel{\circ}{R}$ are nonnegative (resp. positive). The properties and applications of $\stackrel{\circ}{R}$ were studied in [1, pp. 51-52]; $[5,6,19,23,24,41]$, etc.

In the present paper, we prove the vanishing theorem for the kernel ker $\Delta_{S}$ of the Laplacian $\Delta_{S}$ on a closed Riemannian manifold $(M, g)$ with negatively pinched sectional curvature. We find an estimate of its lower eigenvalue depending on the sign of the sectional curvature of $(M, g)$. In addition, we give some applications of the above results.

In conclusion, recall that the Sampson Laplacian $\Delta_{S}$ is of fundamental importance in mathematical physics (e.g., $[11,26,45])$. Note also that the operator $\Delta_{S}$ acting on symmetric covariant 2-tensor fields appears in many problems in Riemannian geometry including Ricci flow (e.g., [3, 9, 16, 21]; [1, pp. 64; 133] and [7, pp. 109-110]). This article continues our study of the Sampson Laplacian, which we carried out in [24].

\section{The Sampson Laplacian and its Weitzenböck curvature operator}

Here, we define the differential operator $\delta^{*}: \mathrm{C}^{\infty}\left(S^{p} M\right) \rightarrow \mathrm{C}^{\infty}\left(S^{p+1} M\right)$ of degree one by the formula $\delta^{*} \varphi=(p+1) \operatorname{Sym}(\nabla \varphi)$ for an arbitrary $\varphi \in \mathrm{C}^{\infty}\left(S^{p} M\right)$ and the standard point-wise symmetry operator Sym : $T^{*} M \otimes S^{p} M \rightarrow S^{p+1} M$. Let $\delta: \mathrm{C}^{\infty}\left(S^{p+1} M\right) \rightarrow \mathrm{C}^{\infty}\left(S^{p} M\right)$ be the adjoint operator for $\delta^{*}$ (see [1, pp. 35, 434]). Then, in accordance with [28], we define the Laplacian

$$
\Delta_{S}=\delta \delta^{*}-\delta^{*} \delta
$$

By [28], $\Delta_{S}$ admits the Weitzenböck decomposition (1.3). In addition, from (1.2) we conclude that $\Re_{p}: S^{p} M \rightarrow$ $S^{p} M$ is a symmetric endomorphism. More properties of the operator $\Delta_{S}$ can be found in the following papers: $[24,30,31,32,33,42]$.

Let $S_{0}^{p} M$ be a vector bundle of traceless symmetric $p$-tensor on $M$, which is defined by the condition trace $_{g} \varphi=0$, where $\operatorname{trace}_{g} \varphi=\sum_{i} \varphi\left(e_{i}, e_{i}, X_{3}, \ldots, X_{p}\right)$ for any $\varphi \in \mathrm{C}^{\infty}\left(S^{p} M\right)$ and an orthonormal basis $\left\{e_{1}, \ldots, e_{n}\right\}$ of $T_{x} M$ at an arbitrary point $x \in M$. Then from (1.2) and (1.3) we conclude that the following proposition is true.

Theorem 2.1. The Sampson Laplacian $\Delta_{S}$ maps the vector space $\mathrm{C}^{\infty}\left(S_{0}^{p} M\right)$ into itself.

Using (1.3), we define the Weitzenböck quadratic form $Q_{p}: S^{p} M \times S^{p} M \rightarrow \mathbb{R}$ by the equality

$$
Q_{p}(\varphi)=g\left(\Re_{p}(\varphi), \varphi\right)=R_{i j} \varphi^{i i_{2} \ldots i_{p}} \varphi_{i_{2} \ldots i_{p}}^{j}+(p-1) R_{i j k l} \varphi^{i l i_{3} \ldots i_{p}} \varphi_{i_{3} \ldots i_{p}}^{j k}
$$

(see also [34]) for local components $\varphi_{i_{1}, \ldots, i_{p}}, R_{i j}$ and $R_{i j k l}$ of an arbitrary $\varphi_{x} \in S_{0}^{p}\left(T^{*} M\right)$, the Ricci tensor Ric and the Riemann curvature tensor $\mathrm{Rm}$, respectively, and for any $\varphi \in S^{p} M$.

Next we prove two propositions on the quadratic form (2.1).

In the paper, we consider positive numbers $\delta>\varepsilon>0$.

Theorem 2.2. Let $\Delta_{S}: \mathrm{C}^{\infty}\left(S_{0}^{2} M\right) \rightarrow \mathrm{C}^{\infty}\left(S_{0}^{2} M\right)$ be the Sampson Laplacian acting on $\mathrm{C}^{\infty}$-sections of the bundle $S_{0}^{2} M$ of traceless symmetric 2-tensors on an $n$-dimensional $(n \geq 2)$ closed Riemannian manifold with negative sectional 
curvature. If $-\delta$ and $-\varepsilon$ are the minimum and maximum of the sectional curvature, then its Weitzenböck quadratic form $Q_{2}(\varphi)$ satisfies the inequalities

$$
-n \delta\|\varphi\|^{2} \leq Q_{2}(\varphi) \leq-n \varepsilon\|\varphi\|^{2}
$$

for any $\varphi \in \mathrm{C}^{\infty}\left(S_{0}^{2} M\right)$.

Proof. First, we consider (2.1) for $p=2$. Thus, for any point $x \in M$ and any $\varphi \in \mathrm{C}^{\infty}\left(S_{0}^{2} M\right)$ there exists an orthonormal eigen-frame $e_{1}, \ldots, e_{n}$ of $T_{x} M$ such that $\varphi_{x}\left(e_{i}, e_{j}\right)=\mu_{i} \delta_{i j}$, where $\delta_{i j}$ is the Kronecker delta, and the following holds (see [1, p. 436]; [3, p. 388]):

$$
Q_{2}(\varphi)=g\left(\Re_{2}\left(\varphi_{x}\right), \varphi_{x}\right)=2 \sum_{i<j} \sec \left(e_{i} \wedge e_{j}\right)\left(\mu_{i}-\mu_{j}\right)^{2} .
$$

Here, sec $\left(e_{i} \wedge e_{j}\right)=R\left(e_{i}, e_{j}, e_{i}, e_{j}\right)$ is the sectional curvature sec $\sigma_{x}$ of $(M, g)$ in the direction of the tangent twoplane section $\sigma_{x}=\operatorname{span}\left\{e_{i}, e_{j}\right\}$ of $T_{x} M$ at $x \in M$. If, in addition, there is a point $x \in M$, where the sectional curvature of $(M, g)$ satisfies the inequalities

$$
-\delta \leq \sec (x) \leq-\varepsilon
$$

for all 2-planes $\pi(x) \subset T_{x} M$ and some constants $\delta>\varepsilon>0$, then from

$$
R_{i j} \varphi^{i k} \varphi_{k}^{j}+R_{i j k l} \varphi^{i l} \varphi^{j k}=2 \sum_{i<j} \sec \left(e_{i} \wedge e_{j}\right)\left(\mu_{i}-\mu_{j}\right)^{2}
$$

we obtain the double inequalities (see [27])

$$
-n \delta\left\|\varphi_{x}\right\|^{2} \leq R_{i j} \varphi^{i k} \varphi_{k}^{j}+R_{i j k l} \varphi^{i l} \varphi^{j k} \leq-n \varepsilon\left\|\varphi_{x}\right\|^{2},
$$

where $\left\|\varphi_{x}\right\|^{2}=\varphi^{i j} \varphi_{i j}$ for the local components $\varphi_{i j}$. In this case, from (2.2) we conclude that the quadratic form $Q_{2}\left(\varphi_{x}\right)$ is negative definite for all nonzero $\varphi_{x} \in S_{0}^{2}\left(T_{x}^{*} M\right)$. In particular, the equality $Q_{2}\left(\varphi_{x}\right)=0$ holds if and only if $\varphi_{x}=0$.

Suppose that $(M, g)$ is compact with negative sectional curvature and denote by $-\delta$ and $-\varepsilon$ the minimum and maximum of its sectional curvature of $(M, g)$. Then from (2.1) we conclude that the Weitzenböck quadratic form satisfies the inequalities

$$
-n \delta\|\varphi\|^{2} \leq Q_{2}(\varphi) \leq-n \varepsilon\|\varphi\|^{2}
$$

for any $\varphi \in \mathrm{C}^{\infty}\left(S_{0}^{2} M\right)$.

Next we will consider the case when $p \geq 3$. At the same time, let $x \in M$ be a point where the sectional curvature of $(M, g)$ satisfies the inequalities

$$
-\delta \leq \sec \pi(x) \leq-\varepsilon<0
$$

for all 2-plans $\pi(x) \subset T_{x} M$. We rewrite the double inequalities (2.2) in the form

$$
-n \delta\left\|\varphi_{x}\right\|^{2}-R_{i j} \varphi^{i k} \varphi_{k}^{j} \leq R_{i j k l} \varphi^{i l} \varphi^{j k} \leq-n \varepsilon\left\|\varphi_{x}\right\|^{2}-R_{i j} \varphi^{i k} \varphi_{k}^{j},
$$

where by [4, p. 81-82] the following inequalities hold:

$$
-(n-1) \delta\left\|\varphi_{x}\right\|^{2} \leq R_{i j} \varphi^{i k} \varphi_{k}{ }_{k} \leq-(n-1) \varepsilon\left\|\varphi_{x}\right\|^{2} .
$$

Then from (2.2) and (2.3) we obtain the following double inequalities:

$$
(-n \delta+(n-1) \varepsilon)\left\|\varphi_{x}\right\|^{2} \leq R_{i j k l} \varphi^{i l} \varphi^{j k} \leq(-n \varepsilon+(n-1) \delta)\left\|\varphi_{x}\right\|^{2} .
$$

From the above we conclude that the following inequalities are satisfied:

$$
(p-1)(-n \delta+(n-1) \varepsilon)\left\|\varphi_{x}\right\|^{2} \leq(p-1) R_{i j k l} \varphi^{i l i_{3} \ldots i_{p}} \varphi_{i_{3} \ldots i_{p}}^{j k} \leq(p-1)(-n \varepsilon+(n-1) \delta)\left\|\varphi_{x}\right\|^{2}
$$

(see [4, p. 82]; [13, p. 91]) for local components $\varphi_{i_{1} \ldots i_{p}}$ of $\varphi_{x} \in S_{0}^{p}\left(T^{*} M\right)$ and $\left\|\varphi_{x}\right\|^{2}=\varphi^{i_{1} \ldots i_{p}} \varphi_{i_{1} \ldots i_{p}}$. In turn, from (2.3) we deduce (see [4, p. 82]; [13, p. 90])

$$
-(n-1) \delta\left\|\varphi_{x}\right\|^{2} \leq R_{i j} \varphi^{i i_{2} \ldots i_{p}} \varphi_{i_{2} \ldots i_{p}}^{j} \leq-(n-1) \varepsilon\left\|\varphi_{x}\right\|^{2} .
$$


From (2.4) and (2.5) we obtain

$$
\begin{aligned}
& ((n-1)(p-1) \varepsilon-((p-1) n+(n-1)) \delta)\left\|\varphi_{x}\right\|^{2} \\
& \quad \leq R_{i j} \varphi^{i i_{2} \ldots i_{p}} \varphi_{i_{2} \ldots i_{p}}^{j}+(p-1) R_{i j k l} \varphi^{i l i_{3} \ldots i_{p}} \varphi_{i_{3} \ldots i_{p}}^{j k} \\
& \quad \leq((n-1)(p-1) \delta-((p-1) n+(n-1)) \varepsilon)\left\|\varphi_{x}\right\|^{2} .
\end{aligned}
$$

Suppose now that $(M, g)$ is a closed Riemannian manifold with negative sectional curvature. Denote by $-\delta$ and $-\varepsilon$ the minimum and maximum of the sectional curvatures of $(M, g)$. Based on the above result, we obtain the following.

Theorem 2.3. Let $\Delta_{S}: \mathrm{C}^{\infty}\left(S_{0}^{p} M\right) \rightarrow \mathrm{C}^{\infty}\left(S_{0}^{p} M\right)$ be the Sampson Laplacian acting on $\mathrm{C}^{\infty}$-sections of the bundle $S_{0}^{p} M$ of traceless symmetric $p$-tensors $(p \geq 3)$ on an $n$-dimensional $(n \geq 2)$ closed Riemannian manifold $(M, g)$ with negative sectional curvature. If $-\delta$ and $-\varepsilon$ are the minimum and maximum of the sectional curvature of $(M, g)$, then its Weitzenböck quadratic form satisfies the inequalities

$$
((n-1)(p-1) \varepsilon-(n p-1) \delta)\left\|\varphi_{x}\right\|^{2} \leq Q_{p}(\varphi) \leq((n-1)(p-1) \delta-(n p-1) \varepsilon)\left\|\varphi_{x}\right\|^{2}
$$

for any $\varphi \in \mathrm{C}^{\infty}\left(S_{0}^{p} M\right)$.

Corollary 2.1. Let $(M, g)$ be an $n$-dimensional $(n \geq 2)$ closed Riemannian manifold with negative sectional curvature and $-\delta$ and $-\varepsilon$ are the minimum and maximum of the sectional curvature, then its curvature operator $\stackrel{\circ}{R}: S_{0}^{2}(M) \rightarrow$ $S_{0}^{2}(M)$ satisfies the inequalities

$$
(-n \delta+(n-1) \varepsilon)\|\varphi\|^{2} \leq g(\stackrel{\circ}{R}(\varphi), \varphi) \leq(-n \varepsilon+(n-1) \delta)\|\varphi\|^{2}
$$

for any $\varphi \in \mathrm{C}^{\infty}\left(S_{0}^{2} M\right)$.

The inequality

$$
(n-1)(p-1) \delta<(n p-1) \varepsilon
$$

implies the condition $Q_{p}\left(\varphi_{x}\right)<0$ for an any nonzero $\varphi_{x} \in S_{0}^{p}\left(T_{x}^{*} M\right)$ at an arbitrary $x \in M$. Side by side, the inequalities $\varepsilon<\delta<0$ and (2.6) can be rewritten in the following form:

$$
1<\delta / \varepsilon<\frac{n p-1}{(n-1)(p-1)}=1+\frac{1}{n-1}+\frac{1}{p-1} .
$$

In this case, the sectional curvature of the manifold $(M, g)$ satisfies the inequalities

$$
-\left(1+\frac{1}{n-1}+\frac{1}{p-1}\right)<-\frac{\delta}{\varepsilon} \leq \frac{\mathrm{sec}}{\delta} \leq-1 .
$$

We can normalize the metric $g$ on the manifold $M$ so that the above double inequalities become

$$
-\left(1+\frac{1}{n-1}+\frac{1}{p-1}\right)<\sec \leq-1 .
$$

Then the following corollary holds.

Corollary 2.2. Let $\Delta_{S}: \mathrm{C}^{\infty}\left(S_{0}^{p} M\right) \rightarrow \mathrm{C}^{\infty}\left(S_{0}^{p} M\right)$ be the Sampson Laplacian acting on $\mathrm{C}^{\infty}$-sections of the bundle $S_{0}^{p} M$ of traceless symmetric $p$-tensors $(p \geq 3)$ on an $n$-dimensional $(n \geq 2)$ closed Riemannian manifold $(M, g)$ with negatively pinched sectional curvature such that (2.7) hold. Then its Weitzenböck quadratic form $Q_{p}(\varphi)$ is negative definite for any $p \geq 2$ and $\varphi \in S_{0}^{p} M$.

On the other hand, the inequalities $\varepsilon<\delta$ and (2.6) can be rewritten in the equivalent form

$$
1-\frac{n+p-2}{n p-1}<\frac{\varepsilon}{\delta}<1 .
$$

Then the sectional curvature of our manifold $(M, g)$ satisfies the inequalities

$$
-1 \leq \frac{\mathrm{sec}}{\delta} \leq-\frac{\varepsilon}{\delta}<-1+\frac{n+p-2}{n p-1} .
$$


We can normalize the metric $g$ on the manifold $(M, g)$ such that the above inequalities become

$$
-1 \leq \sec \leq-\varepsilon<-1+\frac{n+p-2}{n p-1} .
$$

Recall that a Riemannian manifold $(M, g)$, whose sectional curvature satisfies the inequalities

$$
-1 \leq \sec \leq-\varepsilon,
$$

is said to be negatively $\varepsilon$-pinched.

Remark 2.1. More properties of Riemannian manifolds with negatively pinched sectional curvatures can be found, e.g., in [6, 15, 43, 44]. We know from [6, p. 313] that if $(M, g)$ is a locally symmetric manifold with non-constant negative sectional curvature, then its sectional curvature is $1 / 4$-pinched. In our case, we have the pinched sectional curvature with $[-1,-\varepsilon] \subset[-1,-1 / 4]$ such that

$$
-1 \leq \sec \leq-\varepsilon<-1+\frac{n+p-2}{n p-1}<-\frac{1}{4} .
$$

Thus, there are no negative definite Weitzenböck quadratic forms $Q_{p}$ of $\Delta_{S}$ on a Riemannian manifold with negatively 1/4-pinched sectional curvature (see [30, 31, 32]).

On the other hand, M. Gromov and W. Thurston have proved a theorem on negatively $\varepsilon$-pinched Riemannian manifold (see [14]). Namely, for any integer $n \geq 4$ and $\varepsilon \in(0,1)$, there exists a compact Riemannian manifold $(M, g)$ of dimension $n$ such that the sectional curvatures of $(M, g)$ lie in the interval $[-1,-\varepsilon]$, but $(M, g)$ does not admit a metric of constant negative sectional curvature (see [14]). Using this proposition, we obtain the following

Corollary 2.3. There exist closed $n$-dimensional $(n \geq 4)$ Riemannian manifolds $(M, g)$ with negatively pinched sectional curvature, different from compact hyperbolic spaces and such that the Weitzenböck quadratic forms $Q_{p}(\varphi)$ of their Sampson Laplacians $\Delta_{S}: \mathrm{C}^{\infty}\left(S_{0}^{p} M\right) \rightarrow \mathrm{C}^{\infty}\left(S_{0}^{p} M\right)$ are negative definite for any $p \geq 2$.

\section{Vanishing and spectral theorems for Sampson Laplacian}

Let $\Delta_{S}: \mathrm{C}^{\infty}\left(S_{0}^{p} M\right) \rightarrow \mathrm{C}^{\infty}\left(S_{0}^{p} M\right)$ be the Sampson Laplacian acting on $\mathrm{C}^{\infty}$-sections of the bundle $S_{0}^{p} M$ of traceless symmetric $p$-tensors $(p \geq 3)$ on an $n$-dimensional $(n \geq 2)$ compact Riemannian manifold $(M, g)$. Then in accordance with the general theory (e.g., [8]), a real number $\lambda^{(p)}$, for which there is a symmetric $p$-tensor $\varphi \in \mathrm{C}^{\infty}\left(S_{0}^{p} M\right)$ (not identically zero) such that $\Delta_{S} \varphi=\lambda^{(p)} \varphi$, is called an eigenvalue of the Sampson Laplacian $\Delta_{S}: \mathrm{C}^{\infty}\left(S_{0}^{p} M\right) \rightarrow \mathrm{C}^{\infty}\left(S_{0}^{p} M\right)$, and the corresponding symmetric $p$-tensor $\varphi \in \mathrm{C}^{\infty}\left(S_{0}^{p} M\right)$ is called an eigentensor of the Sampson Laplacian $\Delta_{S}$ corresponding to $\lambda^{(p)}$. All nonzero eigentensors corresponding to a fixed eigenvalue $\lambda^{(p)}$ form a vector subspace of $S_{0}^{p} M$ called the eigenspace of the Sampson Laplacian corresponding to its eigenvalue $\lambda^{(p)}$.

Using the general theory of elliptic operators on a closed Riemannian manifold $(M, g)$, it can be proved that $\Delta_{S}$ has a discrete spectrum, denoted by $\operatorname{Spec}^{(p)} \Delta_{S}$, consisting of real eigenvalues of finite multiplicity, which accumulate only at infinity (see also [8]). Moreover, an arbitrary eigenspace of $\Delta_{S}$ is finite-dimensional and the eigentensors corresponding to distinct eigenvalues are orthogonal. In general, the Sampson Laplacian $\Delta_{S}$ is not positive definite and, at the same time, its principal symbol has the form

$$
\sigma\left(\Delta_{S}\right)(\theta, x) \varphi_{x}=-g(\theta, \theta) \varphi_{x}
$$

for $\theta \in T_{x}^{*} M-\{0\}$ and $\varphi_{x} \in S_{0}^{p}\left(T_{x}^{*} M\right)$ at any $x \in M$, but its spectrum satisfies the condition $\operatorname{Spec}^{(p)} \Delta_{S} \subseteq$ $[-C, \infty)$ for some constant $C$ (see $[12$, p. 54]). In this case, we have

$$
\operatorname{Spec}^{(p)} \Delta_{S}=\left\{-\lambda_{1}^{(p)} \leq \ldots \leq-\lambda_{r}^{(p)} \leq 0<\lambda_{r+1}^{(p)} \leq \lambda_{r+2}^{(p)} \leq \ldots \rightarrow \infty\right\} .
$$

Next, we find the conditions for which the spectrum of $\Delta_{S}$ consists of positive numbers.

By direct calculations, we obtain from (1.1) the Bochner-Weitzenböck formula

$$
\frac{1}{2} \Delta_{g}\|\varphi\|^{2}=-g(\bar{\Delta} \varphi, \varphi)+\|\nabla \varphi\|^{2}=-g\left(\Delta_{S} \varphi, \varphi\right)+\|\nabla \varphi\|^{2}-Q_{p}(\varphi)
$$


for an arbitrary $\varphi \in \mathrm{C}^{\infty}\left(S^{p} M\right)$ and the Beltrami Laplacian $\Delta_{g}=\operatorname{div} \circ$ grad, which is defined on $\mathrm{C}^{\infty}$-functions. Let $(M, g)$ be a closed manifold with negative sectional curvature such that (2.8) hold. From (3.1) we deduce the integral equation

$$
\int_{M}\left(\|\nabla \varphi\|^{2}-Q_{p}(\varphi)\right) \mathrm{d}_{g}=0
$$

for $Q_{p}(\varphi)=g\left(\Re_{p}(\varphi), \varphi\right)$ and an arbitrary $\varphi \in \operatorname{ker} \Delta_{L}$. Firstly, we consider the case when $p=2$. In this case, from Theorem 2.2 we know that

$$
Q_{p}(\varphi) \leq-n \varepsilon\|\varphi\|^{2}<0
$$

for an arbitrary nonzero $\varphi \in \mathrm{C}^{\infty}\left(S_{0}^{2} M\right)$. Then from this inequality and (3.2) we conclude that $\varphi \equiv 0$, thus, the kernel of $\Delta_{L}$ is trivial. As a result, we obtain the following vanishing theorem.

Theorem 3.1. Let $\Delta_{S}: \mathrm{C}^{\infty}\left(S_{0}^{2} M\right) \rightarrow \mathrm{C}^{\infty}\left(S_{0}^{2} M\right)$ be the Sampson Laplacian acting on $\mathrm{C}^{\infty}$-sections of the bundle $S_{0}^{2} M$ of traceless symmetric 2-tensors on an $n$-dimensional $(n \geq 2)$ closed Riemannian manifold $(M, g)$ with strictly negative sectional curvature, then $\operatorname{ker} \Delta_{S}$ is trivial.

On the other hand, from (3.1) we deduce the integral inequality

$$
\int_{M} g\left(\Delta_{S} \varphi, \varphi\right) \mathrm{d}_{g} \geq-\int_{M} Q_{2}(\varphi) \mathrm{d}_{g}
$$

for any $\varphi \in \mathrm{C}^{\infty}\left(S_{0}^{2} M\right)$. In addition, if we suppose that a symmetric 2-tensor $\varphi \in \mathrm{C}^{\infty}\left(S_{0}^{2} M\right)$ be an eigentensor of the Sampson Laplacian $\Delta_{S}$ corresponding to $\lambda^{(2)}$, then we can rewrite (3.3) in the following form:

$$
\lambda^{(2)} \int_{M}\|\varphi\|^{2} \mathrm{~d}_{g} \geq n \varepsilon \int_{M}\|\varphi\|^{2} \mathrm{~d}_{g} .
$$

From (3.4) we conclude that $\lambda^{(2)} \geq n \varepsilon>0$. In this case, we have the following

Theorem 3.2. Let $(M, g)$ be an $n$-dimensional $(n \geq 2)$ closed Riemannian manifold with negative sectional curvature. If $-\varepsilon$ is the maximum value of its sectional curvature for some positive number $\varepsilon$, then $\operatorname{Spec}^{(2)} \Delta_{S} \subset[n \varepsilon, \infty)$ for the Sampson Laplacian $\Delta_{S}: \mathrm{C}^{\infty}\left(S_{0}^{2} M\right) \rightarrow \mathrm{C}^{\infty}\left(S_{0}^{2} M\right)$.

Secondary, consider the case when $p \geq 3$. Furthermore, suppose that the inequalities (2.8) are satisfied at any point $x \in M$. In this case, from Theorem 2.3 conclude that if $-\delta$ and $-\varepsilon$ satisfy the inequality

$$
(n-1)(p-1) \delta-(n p-1) \varepsilon<0,
$$

then $Q(\varphi)<0$ for any $\varphi \in \mathrm{C}^{\infty}\left(S_{0}^{p} M\right)$. From the last inequality and the integral equation (3.2) we obtain $\varphi \equiv 0$. Then the following vanishing theorem is true.

Theorem 3.3. Let $\Delta_{S}: \mathrm{C}^{\infty}\left(S_{0}^{p} M\right) \rightarrow \mathrm{C}^{\infty}\left(S_{0}^{p} M\right)$ be the Sampson Laplacian acting on $\mathrm{C}^{\infty}$-sections of the bundle $S_{0}^{p} M$ of traceless symmetric $p$-tensors $(p \geq 3)$ on an $n$-dimensional $(n \geq 2)$ closed Riemannian manifold $(M, g)$ with negative sectional curvature. If $-\delta$ and $-\varepsilon$ are the minimum and maximum of the sectional curvature such that (2.6) are satisfied, then the kernel of $\Delta_{S}$ is trivial.

Taking into account the above and Corollary 2.2, we obtain the following

Corollary 3.1. Let $\Delta_{S}: \mathrm{C}^{\infty}\left(S_{0}^{p} M\right) \rightarrow \mathrm{C}^{\infty}\left(S_{0}^{p} M\right)$ be the Sampson Laplacian acting on $\mathrm{C}^{\infty}$-sections of the bundle $S_{0}^{p} M$ of traceless symmetric $p$-tensors $(p \geq 3)$ on an $n$-dimensional $(n \geq 2)$ closed Riemannian manifold $(M, g)$ with negatively pinched sectional curvature such that (2.7) are satisfied. Then the kernel of $\Delta_{S}$ is trivial.

In addition, taking into account the above and Corollary 2.3, we can formulate the following statement of existence a trivial kernel of the Sampson Laplacian $\Delta_{S}$.

Corollary 3.2. There exist closed $n$-dimensional $(n \geq 4)$ Riemannian manifolds $(M, g)$ with negatively pinched sectional curvature, different from compact hyperbolic spaces and such that the kernels of their Sampson Laplacians $\Delta_{S}: \mathrm{C}^{\infty}\left(S_{0}^{p} M\right) \rightarrow \mathrm{C}^{\infty}\left(S_{0}^{p} M\right)$ are trivial. 
On the other hand, if we suppose that a symmetric $p$-tensor $\varphi \in \mathrm{C}^{\infty}\left(S_{0}^{p} M\right)$ is an eigentensor of $\Delta_{S}$ corresponding to $\lambda^{(p)}$, then we can rewrite (3.2) in the following form:

$$
\lambda^{(p)} \int_{M}\|\varphi\|^{2} \mathrm{~d}_{g} \geq((n-1)(p-1) \varepsilon-(n p-1) \delta) \int_{M}\|\varphi\|^{2} \mathrm{~d}_{g} .
$$

In turn, from (3.5) we conclude that

$$
\lambda^{(p)} \geq(n-1)(p-1) \varepsilon-(n p-1) \delta
$$

In addition, if

$$
(n-1)(p-1) \varepsilon>(n p-1) \delta
$$

for any $p \geq 3$ and $n \geq 2$ then from (3.5) we conclude that $\lambda^{(p)}>0$. As a result, we obtain the following

Theorem 3.4. Let $\Delta_{S}: \mathrm{C}^{\infty}\left(S_{0}^{p} M\right) \rightarrow \mathrm{C}^{\infty}\left(S_{0}^{p} M\right)$ be the Sampson Laplacian acting on $\mathrm{C}^{\infty}$-sections of the bundle $S_{0}^{p} M$ of traceless symmetric $p$-tensors $(p \geq 3)$ on an $n$-dimensional $(n \geq 2)$ closed Riemannian manifold $(M, g)$ with negative sectional curvature. If $-\delta$ and $-\varepsilon$ are the minimum and maximum of the sectional curvature of $(M, g)$, then

$$
\operatorname{Spec}^{(p)} \Delta_{S} \subset[(n-1)(p-1) \varepsilon-(n p-1) \delta, \infty) .
$$

In addition, if (3.6) are satisfied, then the spectrum of $\Delta_{S}$ consists of positive numbers.

\section{Applications to the theory of conformal Killing tensors}

Here, we give some applications of the above results. First, we will consider conformal Killing $p$-forms. Namely, conformal Killing p-forms (or, conformal Killing-Yano $p$-tensors) have been defined on $n$-dimensional Riemannian manifolds $(1 \leq p \leq n-1)$ by S. Tachibana and T. Kashiwada (see [18, 40]) as a natural generalization of conformal Killing vector fields. Since then, these forms were extensively studied by many geometers. These studies were motivated by existence of various applications of conformal Killing $p$-forms (e.g., $[2,37])$.

The vector space of conformal Killing $p$-forms on an $n$-dimensional closed Riemannian manifold $(M, g)$ has a finite dimension $t_{p}(M)$ named the Tachibana number (e.g., $\left.[22,29,35]\right)$. The numbers $t_{1}(M), \ldots, t_{n-1}(M)$ are conformal scalar invariants of $(M, g)$ and satisfy the duality theorem: $t_{p}(M)=t_{n-p}(M)$. The theorem is an analog of the well-known Poincaré duality theorem for the Betti numbers of a closed $(M, g)$. Moreover, we proved in [35] that a) there exist closed Riemannian manifolds with nonzero Tachibana numbers $t_{1}(M), \ldots, t_{n-1}(M)$, b) Tachibana numbers $t_{1}(M), \ldots, t_{n-1}(M)$ are zero for a closed $n$-dimensional $(n \geq 2)$ Riemannian manifold $(M, g)$ with negative curvature operator $\stackrel{\circ}{R}: S_{0}^{2} M \rightarrow S_{0}^{2} M$ defined on the vector bundle $S_{0}^{2} M$. Based on Corollary 2.1, we conclude that if

$$
-1 \leq \sec <-1+1 / n
$$

then the curvature operator $\stackrel{\circ}{R}$ is negative definite. Therefore, the following theorem holds.

Theorem 4.1. If $(M, g)$ is an $n$-dimensional $(n \geq 2)$ closed Riemannian manifold with negatively pinched sectional curvature such that

$$
-1 \leq \sec \leq-1+1 / n,
$$

then its Tachibana numbers $t_{1}(M), \ldots, t_{n-1}(M)$ are equal to zero.

Remark 4.1. The above theorem is a generalization of the following theorem from [43]: Let $(M, g)$ be a closed Riemannian manifold with negatively pinched sectional curvature such that

$$
-1 \leq \sec \leq-\varepsilon .
$$

If the dimension of $M$ is $n=2 m$ (resp., $n=2 m+1)$ and $\varepsilon>1 / 4$ (resp., $\varepsilon>2(m-1) /(8 m-5)$ ), then there are no conformal Killing 2-forms on the manifold. In this case, $t_{2}(M)=t_{n-2}(M)=0$. In addition, the above theorem complements our theorem in [27] on the Tachibana numbers of compact Einstein manifolds. For results on conformally Killing forms on complete non-compact Riemannian manifolds, see [38]. 
Based on the main theorem from [14], we obtain the following.

Corollary 4.1. There exist closed $n$-dimensional $(n \geq 4)$ Riemannian manifolds $(M, g)$ with negatively pinched sectional curvature, different from compact hyperbolic spaces and such that their Tachibana numbers $t_{1}(M), \ldots, t_{n-1}(M)$ are equal to zero.

Next, we consider a conformal Killing symmetric p-tensor $(p \geq 2)$ that is a symmetric trace-free $p$-tensor $\varphi \in \mathrm{C}^{\infty}\left(S_{0}^{p} M\right)$ satisfying the following condition: the trace-free part of $\delta^{*} \varphi$ equals to zero, which is equivalent to the following equation (see [10]; [39, p. 559])

$$
\frac{1}{p+1} \delta^{*} \varphi=-\frac{p}{n+2(p-1)} g \circ \delta \varphi .
$$

In local coordinates, (4.2) can be rewritten in the following form (see also [10]):

$$
\nabla_{\left(i_{0}\right.} \varphi_{\left.i_{1} i_{2} \ldots i_{p}\right)}=-\frac{p}{n+2(p-1)} g_{\left(i_{0} i_{1}\right.} \delta \varphi_{\left.i_{2} \ldots i_{p}\right)},
$$

where we write $\phi_{\left(i_{0} i_{1} \ldots i_{p}\right)}$ for symmetric part of a tensor $\phi_{i_{0} i_{1} \ldots i_{p}}$. Using the definition of the Sampson Laplacian and based on the formula (4.1), we obtain

$$
\begin{array}{r}
\int_{M} g\left(\Delta_{S} \varphi, \varphi\right) \mathrm{d}_{g}=\frac{1}{(p+1)} \int_{M}\left\|\delta^{*} \varphi\right\|^{2} \mathrm{~d}_{g}-\frac{1}{(p-1)} \int_{M}\|\delta \varphi\|^{2} \mathrm{~d}_{g} \\
=-\frac{n-2(p-2)}{n+2(p-1)} \int_{M}\|\delta \varphi\|^{2} \mathrm{~d}_{g} .
\end{array}
$$

In this case, for any conformal Killing tensor $\varphi \in \mathrm{C}^{\infty}\left(S_{0}^{p} M\right)$ we defive the integral formula

$$
\frac{n-2(p-2)}{n+2(p-1)} \int_{M}\|\delta \varphi\|^{2} \mathrm{~d}_{g}+\int_{M}\left(\|\nabla \varphi\|^{2}-Q_{p}(\varphi)\right) \mathrm{d}_{g}=0 .
$$

Using (4.2) and based on Corollary 2.1, we obtain the following proposition.

Corollary 4.2. There exist closed $n$-dimensional $(n \geq 4)$ Riemannian manifolds $(M, g)$ with negatively pinched sectional curvature and different from compact hyperbolic spaces, which have no nonzero symmetric conformal Killing $p$-tensors for any $p \geq 2$.

Remark 4.2. This corollary completes the vanishing theorem in [36] on conformally Killing symmetric tensors of order 2 on a compact Riemannian manifold and its generalization in the case of conformally Killing symmetric tensors of order $p \geq 2$ from $[9,16]$.

\section{References}

[1] Becce A.: Einstein manifolds, Springer-Verlag, Berlin, (1987).

[2] Benn M. and Charlton P.: Dirac symmetry operators from conformal Killing-Yano tensors. Class. Quantum Grav. 14, 1037-1042 (1997).

[3] Berger M. and Ebine D.: Some decomposition of the space of symmetric tensors of a Riemannian manifold. Journal of Differential Geometry. 3,379-392 (1969).

[4] Bochner S. and Yano K.: Curvature and Betti numbers, Princeton Univ. Press, Princeton, (1953).

[5] Bouguignon J.-P.: Formules de Weitzenböck en dimension 4, Geometrie riemannienne en dimension 4. Semin. Arthur Besse, Paris 1978/79, Textes Math., Cedic, Paris. 3, 308-333( 1981).

[6] Burns K. and Katok A.: Manifolds with non-positive curvature. Ergodic Theory of Dynamical Systems. 5:2, 307-317 (1985).

[7] Chow B., Lu P. and Ni L.: Hamilton's Ricci flow, Providence, AMS, (2006).

[8] Craioveanu M., Puta M. and Rassias T.M.: Old and new aspects in spectral geometry, Kluwer Academic Publishers, London, (2001).

[9] Dairbekov N.S. and Sharafutdinov V.A.: Conformal Killing symmetric tensors on Riemannian manifolds. Mat. Tr. 13:1, 85-145 (2010).

[10] Eastwood M.: Higher symmetries of the Laplacian. Annals of Mathematics. 161, 1645-1665 (2005).

[11] Gibbons G.W. and Perry M.J.:Quantizing gravitational instantons. Nuclear Physics B. 146,I,90-108 (1978).

[12] Gilkey P.R.: Invariant theory, the heat equation, and the Atiyah-Singer index theorem. CRC Press, Washington, (1995).

[13] Goldberg S.I.: Curvature and homology. Dover Publications, New-York, (1998).

[14] Gromov M. and Thurston W.: Pinching constants for hyperbolic manifolds. Invent. Math.89 , 1-12 (1987).

[15] Hamenstädt U.: Compact manifolds with 1/4-pinched negative curvature. Lectures Notes in Math., 1481. Global Differential Geometry and Global Analysis, Springer-Verlag, Berlin-Heidelberg, 73-78 (1991).

[16] Heil K., Moroianu A. and Semmelmann U.: Killing and conformal Killing tensors. J. Geom. Phys., 106,383-400 (2016). 
[17] Hitchin, N.: A note on vanishing theorems, In: Geometry and Analysis on Manifolds. Progr. Math. 308, 373-382 (2015).

[18] Kashiwada T.: On conformal Killing tensor. Natural. Sci. Rep. Ochanomizu Univ. 19:2, 67-74 (1968).

[19] Kashiwada T.: On the curvature operator of the second kind. Natural Science Report, Ochanomizu University. 44:2, 69-73 (1993).

[20] Lichnerowicz A.: Propagateurs et commutateurs en relativité générate. Publ. Mathématiques de 1'IHÉS.10:1, 293-344(1961).

[21] Michel R.: Problème d'analyse géomètrique lié à la conjecture de Blaschke. Bull. Soc. Math. France, 101,17-69 (1973).

[22] Mikeš J. and Stepanov S.E.: Betti and Tachibana numbers of compact Riemannian manifolds. Differential Geometry and its Applications. 31:4, 486-495 (2013).

[23] Mikeš J., Sandra I.G. and Stepanov S.E.:On higher order Codazzi tensors on complete Riemannian manifolds. Annals of Global Analysis and Geometry. 56, 429-442 (2019).

[24] Mikeš J., Rovenski V. and Stepanov S.E.: An example of Lichnerowicz-type Laplacian. Annals of Global Analysis and Geometry.58:1, 19-34 (2020).

[25] Petersen P.: Riemannian Geometry. Springer Science, New-York, (2016).

[26] Pilch K. and Schellekens N.: Formulas of the eigenvalues of the Laplacian on tensor harmonics on symmetric coset spaces. J. Math. Phys.25:12, 3455-3459 (1984).

[27] Rovenski V., Stepanov S.E. and Tsyganok I.I.: On the Betti and Tachibana numbers of compact Einstein manifolds. Mathematics. 7:12,1210 (6 pp.) (2019).

[28] Sampson, J.H.: On a theorem of Chern. Trans. AMS. 177, 141-153 (1973).

[29] Stepanov S.E.: Curvature and Tachibana numbers. Sb. Math.202:7, 1059-1069 (2011).

[30] Stepanov S.E. and Mikeš J.: On the Sampson Laplacian. Filomat. 33:4, 1059-1070 (2019).

[31] Stepanov S.E. and Mikeš J.: The spectral theory of the Yano rough Laplacian with some of its applications. Ann. Global Anal. Geom.48:137-46 (2015).

[32] Stepanov S.E. and Shandra I.G.: Geometry of infinitesimal harmonic transformations. Ann. Global Anal. Geom. 24:3, 291-299 (2003).

[33] Stepanov S.E., Tsyganok I.I. and Mikesh J.: On a Laplacian which acts on symmetric tensors. arXiv: 1406.2829 [math.DG].1, 14pp. (2014).

[34] Stepanov S.E.: Fields of symmetric tensors on a compact Riemannian manifold,. Mathematical Notes.52:4, 1048-1050 (1992).

[35] Stepanov S.E. and Tsyganok I.I.:Theorems of existence and of vanishing of conformally killing forms. Russian Mathematics. 58:10, 46-51 (2014).

[36] Stepanov S.E. and Rodionov V.V.: Addition to a work of J.-P. Bourguignon, Differ. Geom. Mnogoobr. Figur, 28 ,68-72 (1997) .

[37] Stepanov S.E.:On conformal Killing 2-form of the electromagnetic field. Journal of Geometry and Physics.33, no. 3-4 , 191-209 (2000).

[38] Stepanov S.E. and Tsyganok I.I.: Conformal Killing $L^{2}$-forms on complete Riemannian manifolds with nonpositive curvature operator. J. of Math. Analysis and Applications. 458:1, 1-8 (2018).

[39] Stephani H., Kramer D., Mac Callum M., Hoenselaers C. and Herlt E.: Exact solutions of Einstein's field equations. Cambridge University Press, (2003).

[40] Tachibana S.: On conformal Killing tensor in a Riemannian space. Tohoku Math. Journal. 21, 56-64 (1969).

[41] Tachibana S. and Ogiue K.: Les variétés riemanniennes dont l'opérateur de coubure restreint est positif sont des sphères d'homologie réelle. C. R. Acad. Sc. Paris.289, 29-30 (1979).

[42] Tandai K. and Sumitomo T.: Killing tensor fields of degree 2 and spectrum of $S O(n+1) / S O(n-1) \times S O(2)$. Osaka J. Math. 17, 649-675 (1980).

[43] Tsagas G.: A relation between Killing tensor fields and negative pinched Riemannian manifolds. Proceedings of the AMS, 22:2, 476-478 (1969).

[44] Vasy A. and Wunsch J.: Absence of super-exponentially decaying eigenfunctions on Riemannian manifolds with pinched negative curvature. Mathematical Research Letters.12:5, 673-684 (2005).

[45] Warner N.P.: The spectra of operators on $C P^{n}$. Proc. R. Soc. Lond. A. 383, 217-230 (1982).

\section{Affiliations}

\section{VLADIMIR ROVENSKI}

AdDRESS: Department of Mathematics, University of Haifa, Mount Carmel, 3498838 Haifa, Israel

E-MAIL: vrovenski@univ.haifa.ac.il

ORCID ID:0000-0003-0591-8307

\section{SERGEY STEPANOV}

AdDREss: Department of Mathematics, Finance University, 49-55, Leningradsky Prospect, 125468 Moscow, Russia

E-MAIL: s.e.stepanov@mail.ru

ORCID ID: 0000-0003-1734-8874

IRINA TSYGANOK

ADDRESS: Department of Mathematics, Finance University, 49-55, Leningradsky Prospect, 125468 Moscow, Russia

E-MAIL: i.i.tsyganok@mail.ru

ORCID ID: 0000-0001-9186-3992 